\title{
Imagem no trauma de face
}

\author{
Imaging in facial trauma
}

Natally de Souza Maciel Rocha1, Juliano Ribeiro Andrade², Shri Krishna Jayanthi

Rocha NSM, Andrade JR, Jayanthi SK. Imagem no trauma de face / Imaging in facial trauma. Rev Med (São Paulo). 2011 out.-dez.;90(4):169-73.

RESUMO: O trauma é uma importante causa de morbimortalidade em todo o mundo e, nesse contexto, o trauma de face é considerado uma das lesões mais devastadoras, devido às possíveis lesões encefálicas e às consequências emocionais relacionadas a deformidades estéticas. Os principais mecanismos de trauma envolvidos nessas lesões são acidentes automobilístico, seguido de agressões, queda de altura e acidentes esportivos. A tomografia computadorizada, amplamente utilizada no trauma, tornou-se o método de imagem de escolha na avaliação desses pacientes, sendo importante na identificação precisa das estruturas comprometidas, orientando a melhor abordagem terapêutica. As diversas formas de apresentação do trauma de face vêm se mostrando verdadeiros desafios na reconstrução funcional e estética desses pacientes, logo, a boa comunicação entre os diversos profissionais envolvidos no tratamento desses pacientes é essencial, especialmente entre radiologistas e cirurgiões. Os objetivos desta revisão são descrever os principais tipos de fraturas de face e discorrer sobre os achados de imagem mais relevantes no seu tratamento.

DESCRITORES: Trauma; Face; Fraturas cranianas; Fraturas orbitárias.

\begin{abstract}
Trauma is an important cause of morbidity and mortality around the world. Facial trauma is considered one of the most devastating lesions seen in trauma centers due to the possibility of brain lesions and the emotional consequences related to facial deformities. The most common mechanism that produces facial fractures is motor vehicle accident, followed by other blunt traumas like falls, aggression and sport accidents. Computed tomography (CT) has become the imaging standard of reference in evaluating these injuries and then give support for the clinicians and surgeons to approach such injuries in a logical and systematic fashion. The many presentations of facial trauma have proven to be real challenges in the functional and aesthetic reconstruction of these patients and so, good communication between the various professionals involved in the treatment of these patients is essential, especially between radiologists and surgeons. The objective of this review is to describe the main types of facial fractures and to discuss the most relevant imaging findings for the best choice of treatment.
\end{abstract}

KEYWORDS: Trauma; Face; Skull fractures; Orbital fractures.

\footnotetext{
${ }^{1}$ Médica residente do $3^{\circ}$ ano de Radiologia do Departamento de Radiologia do Hospital das Clínicas da Faculdade de Medicina da Universidade de São Paulo.

${ }^{2}$ Médico assistente do Setor de Radiologia de Emergência do Hospital das Clínicas da Faculdade de Medicina da Universidade de São Paulo.

${ }^{3}$ Médico chefe de Setor de Radiologia de Emergência do Hospital das Clínicas da Faculdade de Medicina da Universidade de São Paulo.
}

Endereço para correspondência: InRad-HCFMUSP. Av. Dr. Enéas de Carvalho Aguiar, 255, São Paulo, SP, CEP: 05403001. e-mail: skjyanthi@yahoo.com.br 


\section{INTRODUÇÃO}

O trauma é uma importante causa de morbimortalidade em todo o mundo e nesse contexto, o trauma de face é considerado uma das lesões mais devastadoras, devido às possíveis lesões encefálicas e às consequências emocionais relacionadas a deformidade estética. Os principais mecanismos de trauma envolvidos são acidentes automobilístico, seguido de agressões, queda de altura e acidentes esportivos ${ }^{1}$. Sendo assim, o grupo de risco é composto principalmente por homens na faixa etária laborativa, repercutindo tanto no sistema trabalhista nacional quanto no sistema de saúde, uma vez que parte desses pacientes necessita de intervenções multidisciplinares.

O traumatismo na região da face pode afetar desde a pele, planos gordurosos, musculares, neurovasculares, ósseos e até estruturas encefálicas, dependendo da energia cinética envolvida no trauma. O exame clínico associado a estudos radiológicos possue importância ímpar no manejo desses pacientes. A avaliação por radiografia simples é feita essencialmente pelas incidências mento-naso (Waters) e fronto-naso (Caldwell), que muitas vezes já identificam traços de fratura e desalinhamentos. No entanto, a radiografia simples é limitada devido a intensa interposição óssea e de partes moles, causada pelo edema e hemorragia secundários ao trauma ${ }^{2}$. Neste contexto, a tomografia computadorizada (TC) vem se tornando o método de imagem preconizado na avaliação do paciente com fratura de face, sendo importante não apenas no diagnóstico, mas na avaliação da sua extensão tridimensional da fratura, otimizando a terapêutica ${ }^{3}$.

Os estudos radiológicos devem responder as duas principais questões: (a) se as fraturas envolvem estruturas que afetam o funcionamento fisiológico dos seios paranasais, boca, órbita e nariz e (b) se as fraturas resultam em deformidades visíveis. E para que essas perguntas sejam adequadamente respondidas é preciso avaliar: os ossos envolvidos, número de fragmentos, se há afundamento ou desalinhamento dos fragmentos ósseos, o grau de envolvimento de partes moles adjacentes, se há corpos estranhos e avaliar se há lesões encefálicas associadas.

Os objetivos desta revisão são descrever os principais tipos de fraturas de face, incluindo, fraturas nasais, mandibulares, transfaciais (Le Fort), do complexo zigomaticomaxilar e orbitárias, bem como discorrer sobre os achados de imagem mais relevantes na terapêutica dessas fraturas.

\section{FRATURA NASAL}

É a fratura mais comum da face, uma vez que o osso nasal requer menor energia cinética para ser fraturado. O exame clínico é o padrão-ouro para seu diagnóstico, onde se observa crepitação e dor à palpação, no entanto, a avaliação por imagem é importante para determinar a complexidade da fratura e sua extensão, bem como o grau de comprometimento de partes moles adjacentes ${ }^{4}$. Os principais achados que devem ser descritos no relatório são: a presença de fratura dos ossos nasais, do processo frontal da maxila e do septo nasal, bem como se as fraturas são simples ou cominutivas e se há desalinhamentos e suas direções (Figura 1).

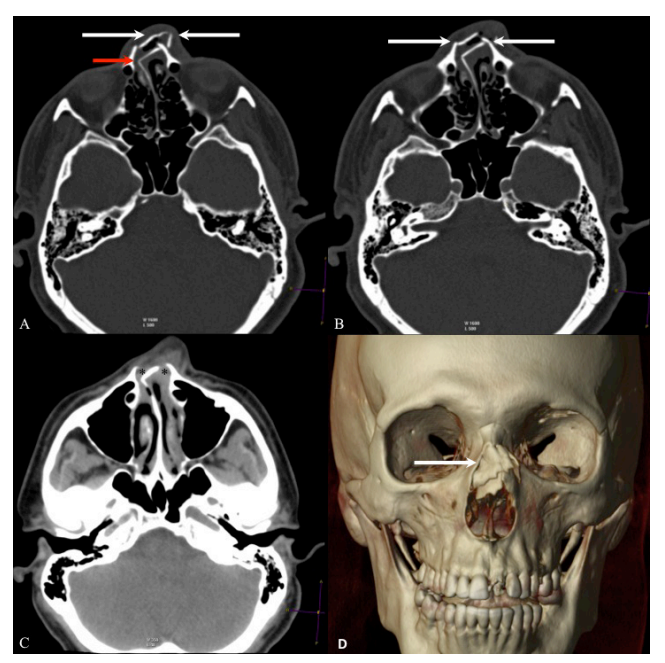

Figura 1. Fratura nasal. (A) TC imagem axial. Fratura dos ossos nasais (setas brancas) e do aspecto anterior do septo nasal (seta vermelha), com encurvamento e desvio para a esquerda dos fragmentos. (B) TC imagem axial. Fratura do processo frontal dos maxilares, com leve desvio para a direita à esquerda e desviado para a esquerda à direita (setas brancas). (C) TC imagem axial. Obliteração da porção anterior das cavidades nasais (asteriscos pretos). (D) TC em reformatação coronal em volume rendering 3D. Fratura nasal com acentuado desvio para a esquerda

Há controvérsias a respeito do manejo das fraturas do nariz, sendo inúmeras abordagens relatadas, como redução manual ou associada a fórceps, septoplastia e até rinosseptoplastia, dependendo do grau de deformidade. O consenso que existe, obedece aos princípios gerais das fraturas, com redução e reposicionamento dos fragmentos fraturados na posição anatômica e imobilização durante o período de consolidação. As fraturas nasais tratadas de forma inadequada resultam em deformidade estética e distúrbios funcionais importantes. Sendo assim, o manejo adequado das mesmas é essencial na recuperação estética e, sobretudo, no restabelecimento da função respiratória.

\section{FRATURA MANDIBULAR}

A fratura mandibular é a segunda mais fre- 
quente no trauma de face, acometendo em ordem decrescente as seguintes estruturas: côndilo, ângulo, corpo, sínfise/parassínfise, ramo e processo coronóide. As fraturas mandibulares podem ser divididas em: (1) simples, fraturas lineares, sem comunicação com a cavidade oral ou pele; (2) compostas, as que apresentam extensão para cavidade oral ou pele; (3) cominutivas, aquelas com múltiplos fragmentos e (4) complicadas, aquelas que comprometem estruturas vasculares e/ou nervosas ${ }^{6}$ (Figura 2).

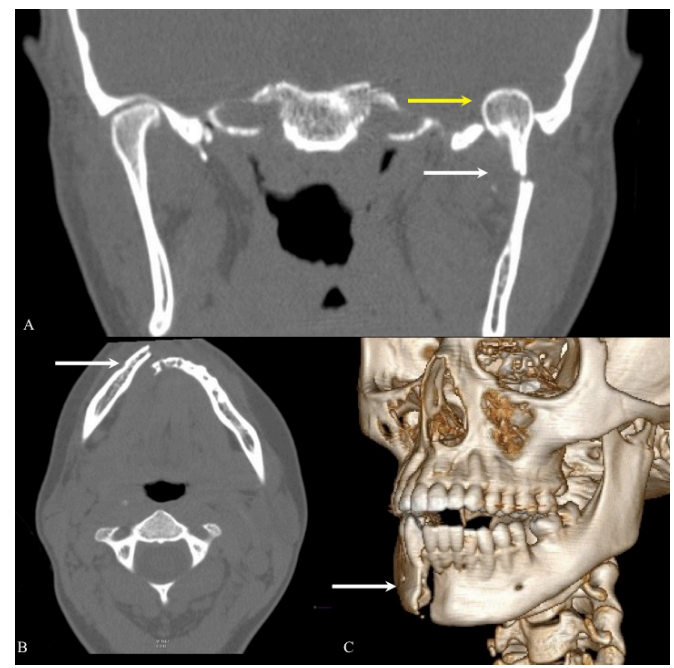

Figura 2. Fratura mandibular. (A) TC reformatação coronal. Fratura do processo condilar esquerdo (seta branca), com herniação superior do côndilo mandibular para a fossa craniana média (seta amarela). (B) TC axial. Fratura do corpo da mandíbula com desalinhamento. (C) TC em reformatação sagital oblíqua em volume rendering 3D. Fratura do corpo da mandíbula com desalinhamento e afastamento dos fragmentos

Os principais achados que devem ser descritos nas fraturas de mandíbula são: localização das fraturas, número de fragmentos e se os mesmos encontram-se desviados, bem como envolvimento da articulação temporomandibular e do canal alveolar inferior. A maioria das fraturas necessita de tratamento cirúrgico, mas o tratamento conservador deve ser considerado, principalmente nas fraturas condilares, se o paciente mantiver oclusão dentária adequada, amplitude de movimento satisfatória e apresentar mínima dor.

\section{FRATURA TRANSFACIAL (LE FORT)}

René Le Fort descreveu os padrões clássicos de fraturas de acordo com seus experimentos realizados com os crânios de cadáveres submetidos a forças de diferentes amplitudes e direções. Estas fraturas envolvem a separação da maxila e da base do crânio, invariavelmente envolvendo as placas pterigóides? Existem três tipos predominantes (Quadro 1):

\section{QUADRO 1. Avaliação das fraturas de Le Fort}

\begin{tabular}{|l|}
\hline Le Fort - O que procurar: \\
\hline 1. Fratura do processo pterigóide - sempre presente \\
nas fraturas de Le Fort \\
2. Fratura do aspecto lateral da fossa nasal - Le Fort I \\
3. Fratura do assoalho da órbita - Le Fort II \\
4. Fratura do arco zigomático - Le Fort III \\
\hline
\end{tabular}

Fonte: Adaptado de How to simplify the CT diagnosis of Le Fort fractures. Rhea JT, Novelline RA. AJR. 2004; 185: 1700-5.

$>$ Le Fort I (fratura maxilar transversa ou horizontal): linha de fratura no plano transmaxilar, acima dos ápices dos dentes e abaixo da junção zigomaticomaxilar. Resulta num aspecto de "palato flutuante" (Figura 3);

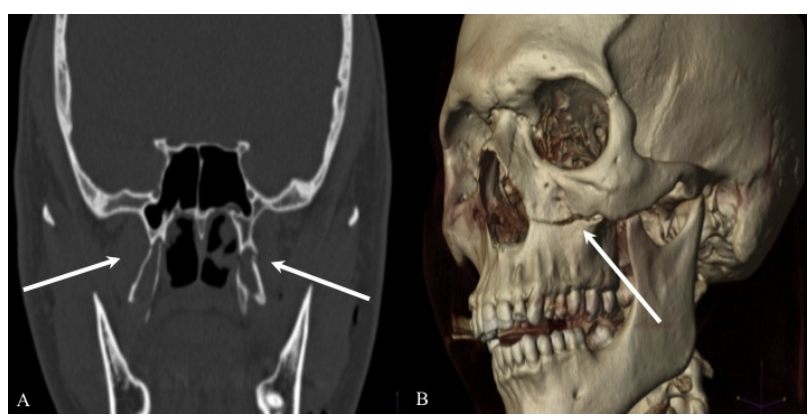

Figura 3. Le Fort I. (A) TC reformatação coronal. Fratura do processo pterigóide bilateral (setas brancas). (B) TC reformatação em volume rendering $3 \mathrm{D}$ sagital oblíquo. Fratura transmaxilar (seta branca)

$>$ Le Fort II (fratura piramidal): linha de fratura no plano subzigomático, a partir da ponte nasal, através do processo frontal da maxila, osso lacrimal e assoalho da órbita, estendendo-se inferiormente através da parede anterior do seio maxilar sob o zigoma. Resulta num aspecto de "maxila flutuante" (Figura 4).

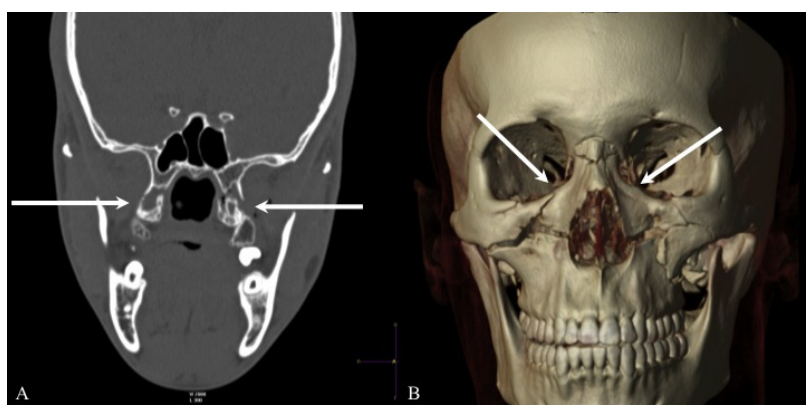

Figura 4. Le Fort II. (A) TC reformatação coronal. Fratura bilateral do processo pterigóide (setas brancas). (B) TC reformatação oblíqua em volume rendering 3D. Fratura do assoalho da órbita (setas brancas) 
$>$ Le Fort III (fratura transversal ou disjunção craniofacial): a linha de fratura percorre o processo nasofrontal, maxilofrontal, paredes orbitárias e arco zigomático. Resulta num aspecto de "face flutuante" (Figura 5).

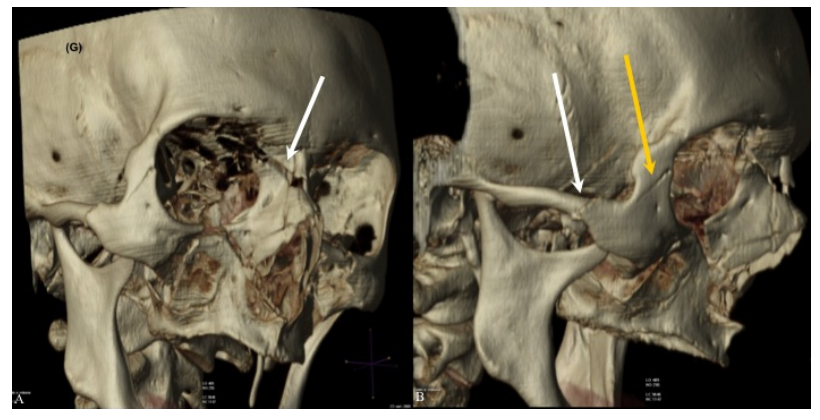

Figura 5. Le Fort III. (A) TC reformatação sagital oblíqua em volume rendering $3 \mathrm{D}$. Fratura do teto da órbita (seta branca). (B) Fratura do arco zigomático (seta branca) e da parede lateral da órbita (seta amarela)

\section{FRATURA EM TRIPÉ}

Também denominada fratura trimalar, é a fratura mais frequente do complexo zigomaticomaxilar e uma das mais encontradas no trauma de face. O principal mecanismo de lesão envolvido é o impacto em direção oblíqua à face, com fraturas de três pontos-chave: (a) sutura frontozigomática; (b) arco zigomático e (c) maxila (parede lateral do seio maxilar) (Figura 6). Deve-se avaliar o alinhamento ósseo, volume orbitário e a integridade do forame infraorbitário, onde se insinua o nervo infraorbital, que é a continuação do nervo maxilar, ramo do trigêmeo'. Este nervo é responsável pela inervação da pele do aspecto superior da bochecha, da mucosa do seio maxilar, dos dentes incisivo, canino e pré-molar, do lábio superior e da pálpebra inferior ${ }^{8}$. As principais indicações cirúrgicas são: fratura cominutiva ou angulada e compressão neural ${ }^{1}$. As complicações mais frequentes são: perda de sensibilidade infraorbitária, déficit de mastigação, oftalmoplegia e deformidade facial.

\section{FRATURAS ORBITÁRIAS}

As fraturas orbitárias podem ser isoladas ou extensão de outras fraturas faciais. A órbita é composta por sete ossos, que possuem a função de proteger os olhos e as estruturas anexas. A porção superior da órbita é composta por estruturas ósseas rígidas, por outro lado, os aspectos medial e inferior da órbita são áreas de maior fragilidade, devido ao fato de serem constituídas por ossos extremamente delgados ${ }^{9}$.

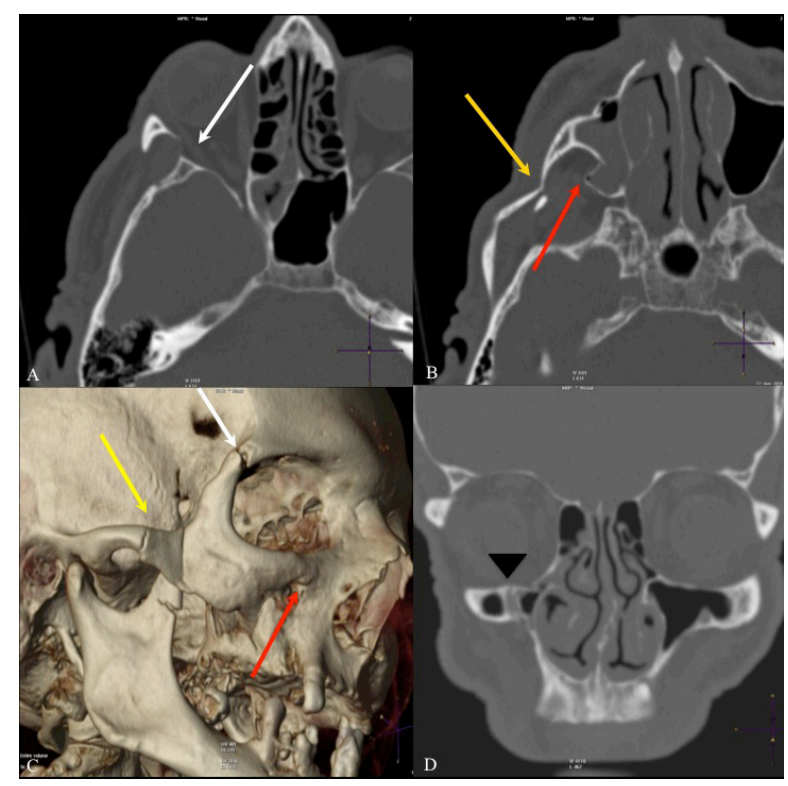

Figura 6. Fratura em tripé. (A) TC imagem axial. Fratura do processo zigomático frontal direito (seta branca). (B) TC reformatação axial oblíqua. Fratura do arco zigomático (seta amarela) e da parede lateral do seio maxilar (seta vermelha). (C) TC reformatação em volume rendering 3D. Visualização tridimensional da fratura em tripé. (D) TC reformatação coronal. Extensão da fratura para o forame infraorbitário (cabeça de seta)

A fratura do tipo blow-out é a mais típica da órbita, sendo decorrente de trauma fechado direito sobre o globo ocular, resultando em aumento da pressão intraocular, que se transmite para a porção mais frágil da órbita, o assoalho (Figura 7A). Em alguns casos a parede medial também pode ser comprometida.

$\mathrm{Na}$ fratura orbitária é importante avaliarmos nos métodos de imagem sinais de herniação dos componentes orbitários, posicionamento do cristalino, que pode estar luxado ou subluxado, pesquisar corpos estranhos, se há hemorragias intra e extraoculares, bem como avaliar as veias oftálmicas e o nervo óptico.

As principais indicações cirúrgicas são: enoftalmia maior que 2,0 mm nas primeiras seis semanas, diplopia significativa, desalinhamento importante, compressão de estruturas neurovasculares e defeito do assoalho orbitário maior do que 1,0 $\mathrm{cm}^{1}$.

As complicações mais frequente são herniação da gordura extraconal e aprisionamento muscular (Figura 7B), causando dificuldade na motilidade ocular. Como complicação mais temida, temos a amaurose e enucleação. A longo prazo pode haver atrofia da gordura orbitária e consequente enoftalmia. 


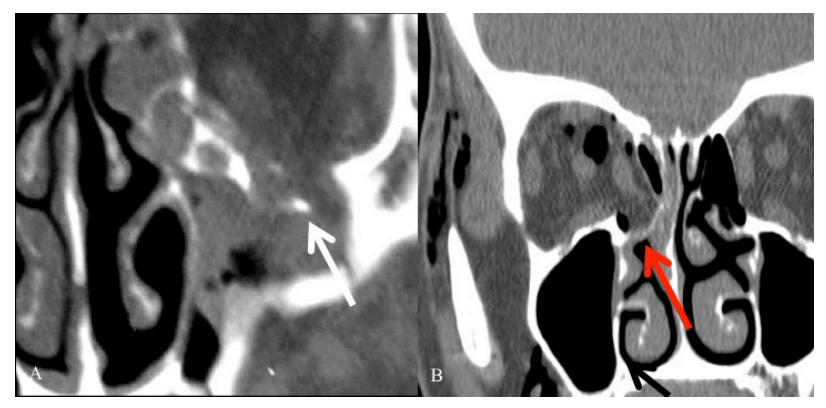

Figura 7. Fratura orbitária blow-out. (A) TC reformatação coronal. Fratura do assoalho orbitário com herniação da gordura intra e extraconal e do músculo reto inferior (seta branca). (B) TC reformatação coronal. Fratura da parede medial e parte do assoalho da órbita com herniação da gordura extraconal e músculo reto lateral (seta vermelha)

\section{REFERÊNCIAS}

1. Hopper RA, Salemy S, Sze RW. Diagnosis of midface fractures with CT: what the surgeon needs to know. Radiographics. 2006;26(3):783-93.

2. Kaur J, Chopra R. Three dimensional CT reconstruction for the evaluation and surgical planning of mid face fractures: a 100 case study. J Maxillofac Oral Surg. 2010;9(4):323-8.

3. Brant-Zawadzki MN, Minagi $\mathrm{H}$, Federle MP, Rowe LD. High resolution CT with image reformation in maxillofacial pathology. AJR Am J Roentgenol. 1982;138(3):477-83.

4. Mohammadi A, Ghasemi-Rad M. Nasal bone fractureultrasonography or computed tomography? Med Ultrasonogr. 2011;13(4):292-5.

\section{CONCLUSÃO}

As fraturas faciais são comuns no contexto de trauma e a TC tem sido amplamente utilizada na avaliação desses pacientes, sendo considerada o método de imagem de escolha nesta avaliação, devido à sua capacidade de demonstrar com precisão as estruturas envolvidas.

As diversas formas de apresentação do trauma facial são um desafio para a reconstrução cirúrgica tanto funcional como estética. Portanto, uma linguagem comum entre os muitos profissionais envolvidos no tratamento desses pacientes é necessária, especialmente entre radiologistas e cirurgiões.

5. Rocha DL, Manganello-Souza LC. Tratamento cirúrgico do trauma bucomaxilofacial. 3a ed. São Paulo: Roca; 2006.

6. Gomes ACA, Silva EDO, Carvalho R, et al. Tratamento das fraturas mandibulares: relato de caso. Rev Cir Traumat Buco-Maxilo-Facial. 2001;1(2):31-8.

7. Rhea JT, Novelline RA. How to simplify the CT diagnosis of le fort fractures. AJR Am J Roentgenol. 2004;184(5):1700-5.

8. Moore K. Anatomia orientada para clínica. 4a ed. Rio de Janeiro: Guanabara Koogan; 2001. p.769.

9. Joseph JM, Glavas IP. Orbital fractures: a review. Clin Ophthalmol. 2011;1(2):95-100. 